\title{
Éditorial: Association canadienne de gérontologie Énoncé de politique: les personnes âgées et les médicaments d'ordonnance
}

Énoncé du problème: Le Canada doit avoir de meilleures politiques pour garantir aux personnes âgées l'accès aux médicaments de manière sûre et efficace.

Portée: Les dépenses pour les médicaments représentent une proportion croissante du coût des soins de santé au Canada, soit 9,9 milliards de dollars en $1993^{2}$ et 14 milliards en 1996. Environ le tiers de l'augmentation des coûts s'explique par l'arrivée de nouveaux médicaments sur le marché et 24 pour cent par l'utilisation plus grande de médicaments par les patients (plus d'ordonnances par personne). ${ }^{3}$ Le partage des frais des médicaments d'ordonnance avec les consommateurs (quote-part, co-assurance, franchise) et le contrôle provincial de la liste des médicaments que le régime public couvrira (le formulaire) sont les deux principales méthodes employées pour contrôler les coûts dans les systèmes de santé provinciaux. ${ }^{4}$ Bien qu'en théorie ces deux méthodes soient censées réduire l'utilisation de médicaments "moins essentiels" (médicaments qui n'améliorent pas beaucoup la santé ou pas plus que d'autres médicaments), en pratique, le partage des frais semble créer des obstacles pécuniaires à l'accès aux médicaments, tant ceux qui sont essentiels que ceux qui le sont moins. ${ }^{5-7}$ Les conséquences de ces deux méthodes de contrôle sur la santé et le bien-être des personnes âgées n'ont pas été assez étudiées au Canada. 8 L'intégrité fiscale du système de santé canadien préoccupe tous les Canadiens, mais les politiques courantes de partage des coûts des médicaments d'ordonnance pénalisent les personnes âgées dont la santé est la plus mauvaise et qui ont la malchance de souffrir de maladies qui coûtent particulièrement cher à soigner.

Analyse: Les personnes âgées font une consommation excessive de certaines classes de médicaments, les plus communs étant les benzodiazépines et d'autres sédatifs hypnotiques non barbituriques pour traiter l'anxiété et l'insomnie, $9-20$ et les anti-inflammatoires non stéroïdiens (AINS) pour traiter les problèmes musculo-squelettiques, principalement l'arthrose. 10,21,22,22-26,26 Cela fait problème parce que les benzodiazépines augmentent le risque de blessures attribuables à des chutes et d'accidents de la route, et peut-être le déclin des facultés cognitives, ${ }^{27-43}$ et les AINS augmentent le risque d'hémorragies gastro-intestinales fatales et non 
fatales.21,44,45,45-57 La surconsommation de médicaments semble reliée à plusieurs facteurs: i) l'inaccessibilité relative d'autres moyens thérapeutiques pour traiter l'anxiété, l'insomnie et les problèmes musculo-squelettiques; ii) l'ignorance des risques des médicaments et du risque d'accoutumance aux benzodiazépines de la part des consommateurs et des médecins; iii) la difficulté d'interrompre la prise de médicaments (résistance des patients, manque de temps des médecins pour examiner leurs dossiers et les éduquer); iv) l'absence de dialogue entre les personnes âgées et les médecins (les médecins croient que les patients s'attendent à se faire prescrire des médicaments et les personnes âgées croient que les médecins prescrivent des médicaments au lieu de consacrer du temps à les écouter parler de leurs problèmes); v) le recours à plusieurs médecins et pharmacies sans qu'un ou l'une d'entre eux ne soit chargé de coordonner l'utilisation des médicaments, et vi) les caractéristiques socio-démographiques des consommateurs-les personnes âgées, les personnes sans travail, les pauvres et les femmes sont plus susceptibles de prendre des médicaments pour l'anxiété et l'insomnie. ${ }^{58-62}$

Certains aspects de la sous-consommation de médicaments n'ont également pas été étudiés à fond (par ex. combien de personnes auraient besoin de prendre des médicaments et n'en prennent pas). Des études faites sur le sujet se dégage une tendance commune. Les médicaments appartenant à certaines classes semblent sous-prescrits aux personnes âgées - thérapie prophylactique, œstrogène de remplacement contre l'ostéoporose, 63 bêtabloquants pour prévenir la récurrence de linfarctus du myocarde ${ }^{64-66}$ et les anti-dépressifs. $67 \mathrm{Il}$ existe deux hypothèses pour expliquer le phénomène. La première est que les personnes âgées sont plus susceptibles de prendre déjà un certain nombre de médicaments et de souffrir de plusieurs maladies et que, pour cela, les médecins hésitent à ajouter un médicament de plus à leur régime médicamenteux déjà complexe, sachant que cela aura un effet négatif sur l'observance thérapeutique. La seconde hypothèse est que le phénomène est attribuable à une attitude partiale par rapport à l'âge qui fait juger le traitement des personnes âgées moins important que celui des jeunes adultes. 62

La prescription de complaisance est un autre sujet qui mérite qu'on s'y arrête. Les personnes âgées sont plus susceptibles de se faire prescrire des médicaments probablement non indiqués; 11 à 46 pour cent d'entre elles reçoivent au moins une ordonnance non indiquée par année. $9,68-78$ Dans les prescriptions de complaisance nous incluons les médicaments qui sont plus ou moins contre-indiqués en raison d'une maladie concomitante (contre-indication médicament-maladie), en raison de la présence d'un médicament dans le régime médicamenteux (interaction médicamentmédicament, médicament qui fait double emploi), de l'âge (médicaments contre-indiqués pour les personnes âgées) ou de la durée du traitement (durée excessive). Les erreurs de prescription représentent à peu près de 19 à 36 pour cent des admissions à l'hôpital pour cause médicamen- 
teuse. 79,80 La coexistence de plusieurs médecins prescripteurs, le nombre de médicaments actuellement commercialisés (plus de 24000), le nombre de contre-indications relatives documentées (plus de 33000) et les lacunes dans les connaissances des médecins attribuables à leur âge et à leur formation sont d'importants facteurs qui contribuent à accroître le risque de la prescription de complaisance.81-83

Le respect adéquat de l'ordonnance par le patient varie de 16 à 73 pour cent. Le non-respect intentionnel ou non de l'ordonnance entraîne des problèmes, ceux des maladies reliées aux médicaments. ${ }^{84}$ Les coûts directs du non-respect des ordonnancés au Canada ont été estimés entre 3,53 et 4,49 milliards de dollars par année. 85 Les facteurs qui influent le plus sur l'observance thérapeutique sont le nombre de médicaments, la complexité du régime médicamenteux, l'utilité perçue du traitement, les effets secondaires et l'oubli. 85 Simplifier le régime médicamenteux, minimiser l'utilisation de médicaments moins essentiels, mieux éduquer les patients, partager la prise de décisions au sujet de l'utilisation des médicaments d'ordonnance et employer des dispositifs permettant d'organiser les médicaments et de rappeler de les prendre peuvent améliorer l'observance thérapeutique. 62

Les personnes âgées sont exclues ou très sous-représentées dans l'évaluation de la sûreté, de l'efficacité et de l'efficience des nouveaux médicaments. ${ }^{1}$ On connaît donc mal les effets des nouveaux médicaments sur elles, particulièrement sur celles qui sont le plus susceptibles d'être exclues des essais - les personnes souffrant d'un certain nombre de maladies qui prennent un plus grand nombre de médicaments. Par conséquent, les médecins ne disposent que de peu d'informations empiriques pour guider leur choix des nouveaux médicaments à prescrire aux personnes âgées en plus mauvaise santé, le groupe qui est précisément celui qui présente le plus grand risque de maladie attribuable aux médicaments.

Recommendations: L'Association canadienne de gérontologie recommande donc que les gouvernements fédéral, provinciaux et territoriaux, en association avec tous les professionnels de la santé et les personnes âgées, travaillent ensemble à élaborer des programmes de meilleures pratiques et d'éducation publique qui viseront la prescription et l'usage sans risque de médicaments.

L'ACG recommande donc que les gouvernements fédéral, provinciaux et territoriaux, en association avec tous les professionnels de la santé, travaillent ensemble à établir des systèmes d'information intégrés qui fourniront aux consommateurs et aux professionnels de la santé des renseignements plus accessibles et à jour sur les médicaments d'ordonnance.

L'ACG recommande donc que les gouvernements fédéral, provinciaux et territoriaux, en association avec les représentants clés des diverses professions de la santé, les personnes âgées et leurs contacts clés, travail- 
lent ensemble à partager l'information pour minimiser les erreurs d'ordonnance.

\section{Note de la rédaction}

On peut se procurer la bibliographie complète auprès de l'Association canadienne de gérontologie (info@cagacg.ca). Seules les principales références sont citées ci-dessous.

\section{Références principales}

Anderson GM, Kerluke KJ, Pulcins IR, Hertzman C, Barer ML. Trends and Determinants of Prescription Drug Expenditures in the Elderly: Data from the British Columbia Pharmacare Program. Inquiry. 1993; 30:199-207.

Cafferata GL, Meyers SM. Pathways to Psychotropic Drugs: Understanding the Basis of Gender Differences. Med Care 1990; 28:285-300.

Col N, Fanale JE, Kronholm P. The Role of Medication Noncompliance and Adverse Drug Reactions in Hospitalizations of the Elderly. Arch.Intern.Med. 1990; 150:841-845.

Gabriel SE, Jaakkimainen L, Bombardier C. Risk for Serious Gastrointestinal Complications Related to Use of Nonsteroidal Anti-inflammatory Drugs. Ann.Intern.Med. 1991; 115:787-796.

Gurwitz J, Col NF, Avorn J. The Exclusion of the Elderly and Women from Clinical Trials in Acute Myocardial Infarction. JAMA 1992; 268(11):1417-1422.2. National Pharmaceutical Strategy Discussion Document. The National Pharmaceutical Strategy Office. Drugs Directorate,Health Protection Branch 1993;

Soumerai SB, Ross-Degnan D, Avorn J, McLaughlin TJ, Choodnovskkiy I. Effects of Medicaid Drug.Payment Limits on Admission to Hospitals and Nursing Homes . N Engl J Med 1991; 325:1072-1077.

Tamblyn RM, McLeod P, Abrahamowicz M, Monette J, Gayton D, Berkson L, et al. Questionable prescribing for elderly patients in Quebec. Can.Med.Assoc.J. 1994; 150 (11):1801-1809.

Tamblyn R, Perreault R. Encouraging the Wise Use of Prescription Medication by Older Adults. In: National Forum on Health, editor. Determinants of Health: Adults and Seniors. Editions MultiMondes, 1998:213-285.

Tinetti ME, Speechley M, Ginter SF. Risk Factors for Falls Among Elderly Persons Living in the Community. N Engl J Med 1988; 319:1701-1707.

Rédigé par Robyn Tamblyn, Ph.D., à la demande du conseil d'administration de l'Association canadienne de gérontologie, l'exposé ci-dessus constitute la position officielle du conseil adoptée en 4 novembre 1999. 